\title{
On the Fekete and Szegö Problem for the Class of Starlike Mappings in Several Complex Variables
}

\author{
Qing-Hua $X u^{1}$ and Tai-Shun Liu ${ }^{2}$ \\ ${ }^{1}$ College of Mathematics and Information Science, Jiangxi Normal University, Nanchang 330022, China \\ ${ }^{2}$ Department of Mathematics, Huzhou Teacher's College, Huzhou 313000, China
}

Correspondence should be addressed to Qing-Hua Xu; xuqh@mail.ustc.edu.cn

Received 22 May 2014; Accepted 7 August 2014; Published 3 September 2014

Academic Editor: Carlos Vazquez

Copyright (c) 2014 Q.-H. Xu and T.-S. Liu. This is an open access article distributed under the Creative Commons Attribution License, which permits unrestricted use, distribution, and reproduction in any medium, provided the original work is properly cited.

Let $\delta$ be the familiar class of normalized univalent functions in the unit disk. Fekete and Szegö proved the well-known result $\max _{f \in \mathcal{S}}\left|a_{3}-\lambda a_{2}^{2}\right|=1+2 e^{-2 \lambda /(1-\lambda)}$ for $\lambda \in[0,1]$. We investigate the corresponding problem for the class of starlike mappings defined on the unit ball in a complex Banach space or on the unit polydisk in $\mathbb{C}^{n}$, which satisfies a certain condition.

\section{Introduction}

Let $\mathscr{A}$ be the class of functions of the form

$$
f(z)=z+\sum_{n=2}^{\infty} a_{n} z^{n}
$$

which are analytic in the open unit disk

$$
\mathbb{U}=\{z \in \mathbb{C}:|z|<1\} .
$$

We denote by $\delta$ the subclass of the normalized analytic function class $\mathscr{A}$ consisting of all functions which are also univalent in $\mathbb{U}$. Let $\mathcal{S}^{*}$ denote the class of starlike functions in $\mathbb{U}$.

It is well known that the Fekete and Szegö inequality is an inequality for the coefficients of univalent analytic functions found by Fekete and Szegö [1], related to the Bieberbach conjecture. Finding similar estimates for other classes of functions is called the Fekete and Szegö problem.

The Fekete and Szegö inequality states that if $f(z)=z+$ $a_{2} z^{2}+a_{3} z^{3}+\cdots \in \mathcal{S}$, then

$$
\max _{f \in \mathcal{S}}\left|a_{3}-\lambda a_{2}^{2}\right|=1+2 e^{-2 \lambda /(1-\lambda)}
$$

for $\lambda \in[0,1]$. After that, there were many papers to consider the corresponding problems for various subclasses of the class $\mathcal{S}$, and many interesting results were obtained. We choose to recall here the investigations by, for example, Kanas [2] (see also [3-5]).
The coefficient estimate problem for the class $\mathcal{S}$, known as the Bieberbach conjecture [6], is settled by de Branges [7], who proved that for a function $f(z)=z+\sum_{k=2}^{\infty} a_{k} z^{k}$ in the class $\mathcal{S}$, then $\left|a_{k}\right| \leq k$, for $k=2,3, \ldots$.

However, Cartan [8] stated that the Bieberbach conjecture does not hold in several complex variables. Therefore, it is necessary to require some additional properties of mappings of a family in order to obtain some positive results, for instance, the convexity and the starlikeness.

In [9], Gong has posed the following conjecture.

Conjecture A. If $f: U^{n} \rightarrow \mathbb{C}^{n}$ is a normalized biholomorphic starlike mapping, where $U^{n}$ is the open unit polydisk in $\mathbb{C}^{n}$, then

$$
\frac{\left\|D^{m} f(0)\left(z^{m}\right)\right\|}{m !} \leq m\|z\|^{m}, \quad z \in U^{n}, m=2,3, \ldots .
$$

In contrast, although the coefficient problem for the class $\mathcal{S}$ had been completely solved, only a few results are known for the inequalities of homogeneous expansions for subclasses of biholomorphic mappings in several complex variables (see, for detail, [9]).

Recently, some best-possible results concerning the coefficient estimates for subclasses of holomorphic mappings in several variables were obtained in work of Graham et al. [10], Graham et al. [11], Hamada et al. [12], Hamada and Honda [13], Kohr [14], X. Liu and T. Liu [15], and Xu and Liu [16] 
In [17], Koepf obtained the following result for $\mathcal{S}^{*}$.

Theorem A. Let $f(z)=z+a_{2} z^{2}+a_{3} z^{3}+\cdots \in \mathcal{S}^{*}$. Then

$$
\left|a_{3}-\lambda a_{2}^{2}\right| \leq \max \{1,|3-4 \lambda|\}, \quad \lambda \in \mathbb{C} .
$$

The above estimation is sharp.

It is natural to ask whether we can extend Theorem A to higher dimensions.

In this paper, we will establish inequalities between the second and third coefficients of homogeneous expansions for starlike mappings defined on the unit ball in Banach complex spaces and the unit polydisc in $\mathbb{C}^{n}$, respectively, which are the natural extension of Theorem A to higher dimensions.

Let $X$ be a complex Banach space with norm $\|\cdot\|$; let $X^{*}$ be the dual space of $X$; let $B$ be the unit ball in $X$. Also, let $\partial U^{n}$ denote the boundary of $U^{n}$, and let $\partial_{0} U^{n}$ be the distinguished boundary of $U^{n}$.

For each $x \in X \backslash\{0\}$, we define

$$
T(x)=\left\{T_{x} \in X^{*}:\left\|T_{x}\right\|=1, T_{x}(x)=\|x\|\right\} .
$$

According to the Hahn-Banach theorem, $T(x)$ is nonempty.

Let $H(B)$ denote the set of all holomorphic mappings from $B$ into $X$. It is well known that if $f \in H(B)$, then

$$
f(y)=\sum_{n=0}^{\infty} \frac{1}{n !} D^{n} f(x)\left((y-x)^{n}\right)
$$

for all $y$ in some neighborhood of $x \in B$, where $D^{n} f(x)$ is the $n$ th-Fréchet derivative of $f$ at $x$, and, for $n \geq 1$,

$$
D^{n} f(x)\left((y-x)^{n}\right)=D^{n} f(x)(\underbrace{y-x, \ldots, y-x}_{n}) \text {. }
$$

Furthermore, $D^{n} f(x)$ is a bounded symmetric $n$-linear mapping from $\prod_{j=1}^{n} X$ into $X$.

A holomorphic mapping $f: B \rightarrow X$ is said to be biholomorphic if the inverse $f^{-1}$ exists and is holomorphic on the open set $f(B)$. A mapping $f \in H(B)$ is said to be locally biholomorphic if the Fréchet derivative $D f(x)$ has a bounded inverse for each $x \in B$. If $f: B \rightarrow X$ is a holomorphic mapping, then $f$ is said to be normalized if $f(0)=0$ and $D f(0)=I$, where $I$ represents the identity operator from $X$ into $X$. Let $\mathcal{S}(B)$ be the set of all normalized biholomorphic mappings on $B$. We say that $f$ is starlike if $f$ is biholomorphic on $B$ and $f(B)$ is starlike with respect to the origin. Let $\mathcal{S}^{*}(B)$ be the set of normalized starlike mappings on $B$.

Suppose that $\Omega \in \mathbb{C}^{n}$ is a bounded circular domain. The first Fréchet derivative and the $m(m \geqslant 2)$-th Fréchet derivative of a mapping $f \in H(\Omega)$ at point $z \in \Omega$ are written by $D f(z), D^{m} f(z)\left(a^{m-1}, \cdot\right)$, respectively. The matrix representations are

$$
D f(z)=\left(\frac{\partial f_{p}(z)}{\partial z_{k}}\right)_{1 \leqslant p, k \leqslant n},
$$

$$
\begin{aligned}
& D^{m} f(z)\left(a^{m-1}, \cdot\right) \\
& \quad=\left(\sum_{l_{1}, l_{2}, \ldots, l_{m-1}=1}^{n} \frac{\partial^{m} f_{p}(z)}{\partial z_{k} \partial z_{l_{1}} \cdots \partial z_{l_{m-1}}} a_{l_{1}} \cdots a_{l_{m-1}}\right)_{1 \leqslant p, k \leqslant n},
\end{aligned}
$$

where $f(z)=\left(f_{1}(z), f_{2}(z), \ldots, f_{n}(z)\right)^{\prime}, a=\left(a_{1}, a_{2}, \ldots, a_{n}\right)^{\prime} \in$ $\mathbb{C}^{n}$.

\section{Some Lemmas}

In order to prove the desired results, we first give some lemmas.

Lemma 1 (see [18]). Let $f: B \rightarrow X$ be a normalized locally biholomorphic mapping. Then $f$ is a starlike mapping on $B$ if and only if

$$
\mathfrak{R} e\left(T_{x}\left(D f(x)^{-1} f(x)\right)\right)>0, \quad x \in B \backslash\{0\}, T_{x} \in T(x) .
$$

Lemma 2. Let $f: U^{n} \rightarrow \mathbb{C}^{n}$ be a normalized locally biholomorphic mapping. Then $f \in \mathcal{S}^{*}\left(U^{n}\right)$ if and only if

$$
\mathfrak{R} e \frac{g_{j}(z)}{z_{j}}>0, \quad z \in U^{n} \backslash\{0\},
$$

where $g(z)=\left(g_{1}(z), g_{2}(z), \ldots, g_{n}(z)\right)^{\prime}=(D f(z))^{-1} f(z)$ and $\left|z_{j}\right|=\|z\|=\max _{1 \leq k \leq n}\left\{\left|z_{k}\right|\right\}$.

Lemma 3 (see [19]). Let $p(z)=1+\sum_{k=1}^{\infty} b_{k} z^{k} \in \mathscr{A}$, and $\mathfrak{R e p}(z)>0, z \in U$; then

$$
\left|b_{2}-\frac{1}{2} b_{1}^{2}\right| \leq 2-\frac{1}{2}\left|b_{1}\right|^{2}
$$

Lemma 4. Suppose that $f \in \mathcal{S}$. Then $F$ defined by $F(x)=$ $\left(f\left(T_{u}(x)\right) / T_{u}(x)\right) x$, where $\|u\|=1$, belongs to $\mathcal{S}^{*}(B)$ if and only if $f \in \mathcal{S}^{*}$.

Proof. Denote $g(x)=f\left(T_{u}(x)\right) / T_{u}(x)$; since $F(x)=g(x) x$, we have

$$
D F(x) \eta=(D g(x) \eta) x+g(x) \eta, \quad \eta \in X .
$$

Straightforward calculation yields

$$
\frac{D g(x) x}{g(x)}=\frac{f^{\prime}\left(T_{u}(x)\right) T_{u}(x)}{f\left(T_{u}(x)\right)}-1 .
$$

It is not difficult to check that

$$
(D F(x))^{-1} \eta=\frac{1}{g(x)}\left[\eta-\frac{(D g(x) \eta) x}{g(x)+D g(x) x}\right], \quad \eta \in X .
$$

Hence

$$
(D F(x))^{-1} F(x)=\frac{f\left(T_{u}(x)\right)}{f^{\prime}\left(T_{u}(x)\right) T_{u}(x)} x .
$$


By using (16), we deduce that

$$
\begin{aligned}
\mathfrak{R} e\left(T_{x}\left((D F(x))^{-1} F(x)\right)\right) & =\mathfrak{R} e\left(\frac{f\left(T_{u}(x)\right)}{f^{\prime}\left(T_{u}(x)\right) T_{u}(x)}\|x\|\right) \\
>0 & \Longleftrightarrow \mathfrak{R} e\left(\frac{\xi f^{\prime}(\xi)}{f^{\prime}(\xi)}\right)>0 .
\end{aligned}
$$

Therefore, by Lemma 1 , we obtain that $F \in \mathcal{S}^{*}(B)$ if and only if $f \in \mathcal{S}^{*}$. This completes the proof of Lemma 4 .

\section{Main Results}

In this section, we state and prove the main results of our present investigation.

Theorem 1. Suppose $f \in \mathcal{S}^{*}(B)$ and

$$
\begin{aligned}
& \frac{1}{2} T_{x}\left(D^{2} f(0)\left(x, \frac{D^{2} f(0)\left(x^{2}\right)}{2 !}\right)\right)\|x\| \\
& =\left(\frac{T_{x}\left(D^{2} f(0)\left(x^{2}\right)\right)}{2 !}\right)^{2}, \quad x \in B, T_{x} \in T(x) .
\end{aligned}
$$

Then

$$
\begin{aligned}
& \left|\frac{T_{x}\left(D^{3} f(0)\left(x^{3}\right)\right)}{3 !\|x\|^{3}}-\lambda\left(\frac{T_{x}\left(D^{2} f(0)\left(x^{2}\right)\right)}{2 !\|x\|^{2}}\right)^{2}\right| \\
& \quad \leq \max \{1,|3-4 \lambda|\}, \quad x \in B, \lambda \in \mathbb{C} .
\end{aligned}
$$

The above estimate is sharp.

Proof. Fix $x \in B \backslash\{0\}$ and denote $x_{0}=x /\|x\|$. Let $p: U \rightarrow \mathbb{C}$ be given by

$$
p(\xi)= \begin{cases}\frac{T_{x_{0}}\left(g\left(\xi x_{0}\right)\right)}{\xi}, & \xi \neq 0, \\ 1, & \xi=0,\end{cases}
$$

where $g(x)=(D f(x))^{-1} f(x)$. Then $p \in \mathscr{A}, p(0)=1$, and

$$
\begin{aligned}
p(\xi)= & +\frac{T_{x_{0}}\left(D^{2} g(0)\left(x_{0}^{2}\right)\right)}{2 !} \xi+\cdots \\
& +\frac{T_{x_{0}}\left(D^{m} g(0)\left(x_{0}^{m}\right)\right)}{m !} \xi^{m-1}+\cdots .
\end{aligned}
$$

Since $f \in \mathcal{S}^{*}(B)$, from Lemma 1 , we have

$$
\mathfrak{R} e(p(\xi))>0, \quad \xi \in U .
$$

In view of Lemma 3 , we obtain that

$$
\begin{aligned}
& \left|\frac{T_{x_{0}}\left(D^{3} g(0)\left(x_{0}^{3}\right)\right)}{3 !}-\frac{1}{2}\left(\frac{T_{x_{0}}\left(D^{2} g(0)\left(x_{0}^{2}\right)\right)}{2 !}\right){ }^{2}\right| \\
& \quad \leq 2-\frac{1}{2}\left|\frac{T_{x_{0}}\left(D^{2} g(0)\left(x_{0}^{2}\right)\right)}{2 !}\right|^{2} .
\end{aligned}
$$

That is,

$$
\begin{aligned}
& \left|\frac{T_{x}\left(D^{3} g(0)\left(x^{3}\right)\right)\|x\|}{3 !}-\frac{1}{2}\left(\frac{T_{x}\left(D^{2} g(0)\left(x^{2}\right)\right)}{2 !}\right)^{2}\right| \\
& \quad \leq 2\|x\|^{4}-\frac{1}{2}\left|\frac{T_{x}\left(D^{2} g(0)\left(x^{2}\right)\right)}{2 !}\right|^{2} .
\end{aligned}
$$

On the other hand, since $g(x)=(D f(x))^{-1} f(x)$, we have

$$
\begin{aligned}
x+ & \frac{D^{2} f(0)\left(x^{2}\right)}{2 !}+\frac{D^{3} f(0)\left(x^{3}\right)}{3 !}+\cdots \\
= & \left(I+D^{2} f(0)(x, \cdot)+\frac{D^{3} f(0)\left(x^{2}, \cdot\right)}{2 !}+\cdots\right) \\
& \times\left(D g(0)+\frac{D^{2} g(0)\left(x^{2}\right)}{2 !}+\frac{D^{3} g(0)\left(x^{3}\right)}{3 !}+\cdots\right) .
\end{aligned}
$$

Comparing with the homogeneous expansion of two sides of the above equality, we obtain

$$
\begin{aligned}
D g(0) x=x, & \frac{D^{2} g(0)\left(x^{2}\right)}{2 !}=-\frac{D^{2} f(0)\left(x^{2}\right)}{2 !}, \\
\frac{D^{3} f(0)\left(x^{3}\right)}{3 !}= & \frac{D^{3} g(0)\left(x^{3}\right)}{3 !}+\frac{D^{3} f(0)\left(x^{3}\right)}{2 !} \\
& -D^{2} f(0)\left(x, \frac{D^{2} f(0)\left(x^{2}\right)}{2 !}\right) .
\end{aligned}
$$

Equation (27) may be rewritten as follows:

$$
\begin{aligned}
- & 2 \frac{D^{3} f(0)\left(x^{3}\right)}{3 !} \\
& =\frac{D^{3} g(0)\left(x^{3}\right)}{3 !}-D^{2} f(0)\left(x, \frac{D^{2} f(0)\left(x^{2}\right)}{2 !}\right) .
\end{aligned}
$$

Thus, from (18) of Theorem 1, (24), (26), and (28), we deduce that

$$
\begin{aligned}
\left|\frac{T_{x}\left(D^{3} f(0)\left(x^{3}\right)\right)\|x\|}{3 !}-\lambda\left(\frac{T_{x}\left(D^{2} f(0)\left(x^{2}\right)\right)}{2 !}\right)^{2}\right| \\
=\mid-\frac{1}{2} \frac{T_{x}\left(D^{3} g(0)\left(x^{3}\right)\right)\|x\|}{3 !} \\
+\frac{1}{2} T_{x}\left(D^{2} f(0)\left(x, \frac{D^{2} f(0)\left(x^{2}\right)}{2 !}\right)\right)\|x\| \\
-\lambda\left(\frac{T_{x}\left(D^{2} f(0)\left(x^{2}\right)\right)}{2 !}\right)^{2} \mid
\end{aligned}
$$




$$
\begin{aligned}
=\frac{1}{2} \mid & -\frac{T_{x}\left(D^{3} g(0)\left(x^{3}\right)\right)\|x\|}{3 !} \\
& +(2-2 \lambda)\left(\frac{T_{x}\left(D^{2} f(0)\left(x^{2}\right)\right)}{2 !}\right)^{2} \mid \\
=\frac{1}{2} \mid & -\frac{T_{x}\left(D^{3} g(0)\left(x^{3}\right)\right)\|x\|}{3 !}+\frac{1}{2}\left(\frac{T_{x}\left(D^{2} f(0)\left(x^{2}\right)\right)}{2 !}\right)^{2} \\
& +\left(\frac{3}{2}-2 \lambda\right)\left(\frac{T_{x}\left(D^{2} f(0)\left(x^{2}\right)\right)}{2 !}\right)^{2} \mid \\
\leq & \frac{1}{2}\left(2\|x\|^{4}-\frac{1}{2}\left|\frac{T_{x}\left(D^{2} g(0)\left(x^{2}\right)\right)}{2 !}\right|^{2}\right. \\
& \left.+\left|\frac{3}{2}-2 \lambda\right|\left|\frac{T_{x}\left(D^{2} g(0)\left(x^{2}\right)\right)}{2 !}\right|^{2}\right) .
\end{aligned}
$$

If now $|\lambda-(3 / 4)| \leq 1 / 4$, then

$$
\begin{gathered}
\mid \frac{T_{x}\left(D^{3} f(0)\left(x^{3}\right)\right)\|x\|}{3 !}-\lambda\left(\frac{T_{x}\left(D^{2} f(0)\left(x^{2}\right)\right)}{2 !}\right) \\
=\frac{1}{2}\left(2\|x\|^{4}-\frac{1}{2}\left|\frac{T_{x}\left(D^{2} g(0)\left(x^{2}\right)\right)}{2 !}\right|^{2}\right. \\
\left.+\frac{1}{2}\left|\frac{T_{x}\left(D^{2} g(0)\left(x^{2}\right)\right)}{2 !}\right|^{2}\right) \leq\|x\|^{4} .
\end{gathered}
$$

On the other hand, if $|\lambda-(3 / 4)| \geq 1 / 4$, then we use $\left|T_{x}\left(D^{2} g(0)\left(x^{2}\right)\right) / 2 !\right| \leq 2\|x\|^{2}$ and get

$$
\begin{aligned}
& \left|\frac{T_{x}\left(D^{3} f(0)\left(x^{3}\right)\right)\|x\|}{3 !}-\lambda\left(\frac{T_{x}\left(D^{2} f(0)\left(x^{2}\right)\right)}{2 !}\right)^{2}\right| \\
& \quad=\|x\|^{4}+\frac{1}{2}\left(\left|\frac{3}{2}-2 \lambda\right|-\frac{1}{2}\right)\left|\frac{T_{x}\left(D^{2} g(0)\left(x^{2}\right)\right)}{2 !}\right|^{2} \\
& \quad \leq\|x\|^{4}+(|3-4 \lambda|-1)\|x\|^{4} \\
& \quad=|3-4 \lambda|\|x\|^{4} .
\end{aligned}
$$

The following example shows that the estimation of Theorem 1 is sharp.

Example. If $|\lambda-(3 / 4)| \geq 1 / 4$, we consider the following example:

$$
f(x)=\frac{x}{\left(1-T_{u}(x)\right)^{2}}, \quad x \in B,\|u\|=1 .
$$

By Lemma 4 , we obtain that $f \in \mathcal{S}^{*}(B)$.
It is not difficult to check that the mapping $f(x)$ satisfies the condition of Theorem 1. Setting $x=r u(0<r<1)$ in (32), we obtain that

$$
\left|\frac{T_{x}\left(D^{3} f(0)\left(x^{3}\right)\right)}{3 !\|x\|^{3}}-\lambda\left(\frac{T_{x}\left(D^{2} f(0)\left(x^{2}\right)\right)}{2 !\|x\|^{2}}\right)^{2}\right|=|3-4 \lambda| .
$$

If $|\lambda-(3 / 4)| \leq 1 / 4$, we consider the following example:

$$
f(x)=\frac{x}{1-\left(T_{u}(x)\right)^{2}}, \quad x \in B,\|u\|=1 .
$$

In view of Lemma 4 , we deduce that $f \in \mathcal{S}^{*}(B)$.

It is not difficult to verify that the mapping $f(x)$ satisfies the condition of Theorem 1. Taking $x=r u(0<r<1)$ in (34), we have

$$
\left|\frac{T_{x}\left(D^{3} f(0)\left(x^{3}\right)\right)}{3 !\|x\|^{3}}-\lambda\left(\frac{T_{x}\left(D^{2} f(0)\left(x^{2}\right)\right)}{2 !\|x\|^{2}}\right)^{2}\right|=1 .
$$

This completes the proof of Theorem 1 .

Remark 2. When $X=\mathbb{C}, B=U$, Theorem 1 is equivalent to Theorem A.

Theorem 3. Suppose $f \in \mathcal{S}^{*}\left(U^{n}\right)$ and

$$
\begin{array}{r}
\frac{1}{2} D^{2} f_{k}(0)\left(z_{0}, \frac{D^{2} f(0)\left(z_{0}^{2}\right)}{2 !}\right) \frac{z_{k}}{\|z\|}=\left(\frac{D^{2} f_{k}(0)\left(z_{0}^{2}\right)}{2 !}\right)^{2}, \\
z \in U^{n},
\end{array}
$$

for $z \in U^{n} \backslash\{0\}$, where $k=1,2, \ldots, n, z_{0}=z /\|z\|$. Then

$$
\begin{gathered}
\left\|\frac{D^{3} f(0)\left(z^{3}\right)}{3 !}-\lambda \frac{1}{2} D^{2} f(0)\left(z, \frac{D^{2} f(0)\left(z^{2}\right)}{2 !}\right)\right\| \\
\leq\|z\|^{3} \max \{1,|3-4 \lambda|\}, \quad z \in U^{n}, \lambda \in \mathbb{C} .
\end{gathered}
$$

The above estimate is sharp.

Proof. For any $z \in U^{n} \backslash\{0\}$, denote $z_{0}=z /\|z\|$. Let $q_{j}: U \rightarrow$ $\mathbb{C}$ be given by

$$
q_{j}(\xi)= \begin{cases}\frac{g_{j}\left(\xi z_{0}\right)\|z\|}{\xi z_{j}}, & \xi \neq 0, \\ 1, & \xi=0,\end{cases}
$$

where $g(z)=(D f(z))^{-1} f(z)$ and $j$ satisfies $\left|z_{j}\right|=\|z\|=$ $\max _{1 \leq k \leq n}\left\{\left|z_{k}\right|\right\}$. Then $q_{j} \in \mathscr{A}, q_{j}(0)=1$, and

$$
\begin{aligned}
q_{j}(\xi)= & 1+\frac{D^{2} g_{j}(0)\left(z_{0}^{2}\right)\|z\|}{2 ! z_{j}} \xi \\
& +\frac{D^{3} g_{j}(0)\left(z_{0}^{3}\right)\|z\|}{3 ! z_{j}} \xi^{2}+\cdots
\end{aligned}
$$


Since $f \in \mathcal{S}^{*}\left(U^{n}\right)$, from Lemma 2, we deduce that $\mathfrak{R} e\left(q_{j}(\xi)\right)>0, \xi \in U$. Therefore, according to Lemma 3, we have

$$
\begin{aligned}
& \left|\frac{D^{3} g_{j}(0)\left(z_{0}^{3}\right)\|z\|}{3 ! z_{j}}-\frac{1}{2}\left(\frac{D^{2} g_{j}(0)\left(z_{0}^{2}\right)\|z\|}{2 ! z_{j}}\right)^{2}\right| \\
& \quad \leq 2-\frac{1}{2}\left|\frac{D^{2} g_{j}(0)\left(z_{0}^{2}\right)\|z\|}{2 ! z_{j}}\right|^{2} .
\end{aligned}
$$

Hence, in view of (26), (28), and (32) of Theorem 3, we obtain that

$$
\begin{aligned}
& \left|\frac{D^{3} f_{j}(0)\left(z_{0}^{3}\right)\|z\|}{3 ! z_{j}}-\lambda \frac{1}{2} D^{2} f_{j}(0)\left(z_{0}, \frac{D^{2} f(0)\left(z_{0}^{2}\right)}{2 !}\right) \frac{\|z\|}{z_{j}}\right| \\
& =\left|\frac{D^{3} f_{j}(0)\left(z_{0}^{3}\right)\|z\|}{3 ! z_{j}}-\lambda\left(\frac{D^{2} f_{j}(0)\left(z_{0}^{2}\right)\|z\|}{2 ! z_{j}}\right)^{2}\right| \\
& =\mid-\frac{1}{2} \frac{D^{3} g_{j}(0)\left(z_{0}^{3}\right)\|z\|}{3 ! z_{j}}+\frac{1}{2} D^{2} f_{j}(0)\left(z_{0}, \frac{D^{2} f(0)\left(z_{0}^{2}\right)}{2 !}\right) \\
& \times \frac{\|z\|}{z_{j}}-\lambda\left(\frac{D^{2} f_{j}(0)\left(z_{0}^{2}\right)\|z\|}{2 ! z_{j}}\right)^{2} \\
& =\left|-\frac{1}{2} \frac{D^{3} g_{j}(0)\left(z_{0}^{3}\right)\|z\|}{3 ! z_{j}}+(1-\lambda)\left(\frac{D^{2} f_{j}(0)\left(z_{0}^{2}\right)\|z\|}{2 ! z_{j}}\right)^{2}\right| \\
& =\frac{1}{2}\left|-\frac{D^{3} g_{j}(0)\left(z_{0}^{3}\right)\|z\|}{3 ! z_{j}}+(2-2 \lambda)\left(\frac{D^{2} f_{j}(0)\left(z_{0}^{2}\right)\|z\|}{2 ! z_{j}}\right)^{2}\right| \\
& =\frac{1}{2}\left|-\frac{D^{3} g_{j}(0)\left(z_{0}^{3}\right)\|z\|}{3 ! z_{j}}+(2-2 \lambda)\left(\frac{D^{2} g_{j}(0)\left(z_{0}^{2}\right)\|z\|}{2 ! z_{j}}\right)^{2}\right| \\
& =\frac{1}{2} \mid-\frac{D^{3} g_{j}(0)\left(z_{0}^{3}\right)\|z\|}{3 ! z_{j}}+\frac{1}{2}\left(\frac{D^{2} g_{j}(0)\left(z_{0}^{2}\right)\|z\|}{2 ! z_{j}}\right)^{2} \\
& +\left(\frac{3}{2}-2 \lambda\right)\left(\frac{D^{2} g_{j}(0)\left(z_{0}^{2}\right)\|z\|}{2 ! z_{j}}\right)^{2} \mid \\
& \leq \frac{1}{2}\left(2-\frac{1}{2}\left|\frac{D^{2} g_{j}(0)\left(z_{0}^{2}\right)\|z\|}{2 ! z_{j}}\right|^{2}\right. \\
& \left.+\left|\frac{3}{2}-2 \lambda\right|\left|\frac{D^{2} g_{j}(0)\left(z_{0}^{2}\right)\|z\|}{2 ! z_{j}}\right|^{2}\right) .
\end{aligned}
$$

If now $|\lambda-(3 / 4)| \leq 1 / 4$, then

$$
\begin{aligned}
& \left|\frac{D^{3} f_{j}(0)\left(z_{0}^{3}\right)\|z\|}{3 ! z_{j}}-\lambda \frac{1}{2} D^{2} f_{j}(0)\left(z_{0}, \frac{D^{2} f(0)\left(z_{0}^{2}\right)}{2 !}\right) \frac{\|z\|}{z_{j}}\right| \\
& \leq \frac{1}{2}\left(2-\frac{1}{2}\left|\frac{D^{2} g_{j}(0)\left(z_{0}^{2}\right)\|z\|}{2 ! z_{j}}\right|^{2}+\frac{1}{2}\left|\frac{D^{2} g_{j}(0)\left(z_{0}^{2}\right)\|z\|}{2 ! z_{j}}\right|^{2}\right) \\
& =1 .
\end{aligned}
$$

On the other hand, if $|\lambda-(3 / 4)| \geq 1 / 4$, then we use $\left|D^{2} g_{j}(0)\left(z_{0}^{2}\right)\|z\| / 2 ! z_{j}\right| \leq 2$ and get

$$
\begin{aligned}
& \left|\frac{D^{3} f_{j}(0)\left(z_{0}^{3}\right)\|z\|}{3 ! z_{j}}-\lambda \frac{1}{2} D^{2} f_{j}(0)\left(z_{0}, \frac{D^{2} f(0)\left(z_{0}^{2}\right)}{2 !}\right) \frac{\|z\|}{z_{j}}\right| \\
& \quad \leq 1+\frac{1}{2}\left(\left|\frac{3}{2}-2 \lambda\right|-\frac{1}{2}\right)\left|\frac{D^{2} g_{j}(0)\left(z_{0}^{2}\right)\|z\|}{2 ! z_{j}}\right|^{2} \\
& \quad=1+|3-4 \lambda|-1=|3-4 \lambda| .
\end{aligned}
$$

Then, by using (42) and (43), we have

$$
\begin{aligned}
& \left|\frac{D^{3} f_{j}(0)\left(z_{0}^{3}\right)\|z\|}{3 ! z_{j}}-\lambda \frac{1}{2} D^{2} f_{j}(0)\left(z_{0}, \frac{D^{2} f(0)\left(z_{0}^{2}\right)}{2 !}\right) \frac{\|z\|}{z_{j}}\right| \\
& \quad \leq \max \{1,|3-4 \lambda|\} .
\end{aligned}
$$

If $z_{0} \in \partial_{0} D^{n}$, then we have

$$
\begin{aligned}
& \left|\frac{D^{3} f_{j}(0)\left(z_{0}^{3}\right)}{3 !}-\lambda \frac{1}{2} D^{2} f_{j}(0)\left(z_{0}, \frac{D^{2} f(0)\left(z_{0}^{2}\right)}{2 !}\right)\right| \\
& \quad \leq \max \{1,|3-4 \lambda|\}, \quad j=1,2, \ldots, n .
\end{aligned}
$$

Also since

$$
\begin{array}{r}
\frac{D^{3} f_{j}(0)\left(z^{3}\right)}{3 !}-\lambda \frac{1}{2} D^{2} f_{j}(0)\left(z, \frac{D^{2} f(0)\left(z^{2}\right)}{2 !}\right), \\
(j=1,2, \ldots, n)
\end{array}
$$

is a holomorphic function on $\bar{U}^{n}$, in view of the maximum modulus theorem of holomorphic function on the unit polydisc, we obtain

$$
\begin{array}{r}
\left|\frac{D^{3} f_{j}(0)\left(z_{0}^{3}\right)}{3 !}-\lambda \frac{1}{2} D^{2} f_{j}(0)\left(z_{0}, \frac{D^{2} f(0)\left(z_{0}^{2}\right)}{2 !}\right)\right| \\
\leq \max \{1,|3-4 \lambda|\}, \quad z_{0} \in \partial U^{n}, j=1,2, \ldots, n .
\end{array}
$$

That is,

$$
\begin{aligned}
& \left|\frac{D^{3} f_{j}(0)\left(z^{3}\right)}{3 !}-\lambda \frac{1}{2} D^{2} f_{j}(0)\left(z, \frac{D^{2} f(0)\left(z^{2}\right)}{2 !}\right)\right| \\
& \quad \leq\|z\|^{3} \max \{1,|3-4 \lambda|\}, \quad z \in U^{n}, j=1,2, \ldots, n .
\end{aligned}
$$


Hence

$$
\begin{aligned}
& \left\|\frac{D^{3} f(0)\left(z^{3}\right)}{3 !}-\lambda \frac{1}{2} D^{2} f(0)\left(z, \frac{D^{2} f(0)\left(z^{2}\right)}{2 !}\right)\right\| \\
& \quad \leq\|z\|^{3} \max \{1,|3-4 \lambda|\}, \quad z \in U^{n} .
\end{aligned}
$$

Finally, in order to see that the estimation of Theorem 3 is sharp, it suffices to consider the following mappings.

If $|\lambda-(3 / 4)| \geq 1 / 4$, we consider the following example:

$$
f(z)=\left(\frac{z_{1}}{\left(1-z_{1}\right)^{2}}, \frac{z_{2}}{\left(1-z_{2}\right)^{2}}, \ldots, \frac{z_{n}}{\left(1-z_{n}\right)^{2}}\right)^{\prime}, \quad z \in U^{n} .
$$

If $|\lambda-(3 / 4)| \leq 1 / 4$, we consider the following example:

$$
f(z)=\left(\frac{z_{1}}{1-z_{1}^{2}}, \frac{z_{2}}{1-z_{2}^{2}}, \ldots, \frac{z_{n}}{1-z_{n}^{2}}\right)^{\prime}, \quad z \in U^{n} .
$$

In view of Problem 6.2.5 of [19], we deduce that the mappings $f(z)$, defined in (50) and (51), are in the class $\mathcal{S}^{*}\left(U^{n}\right)$.

It is not difficult to verify that the mappings $f(z)$ defined in (50) and (51) satisfy the condition of Theorem 3. Taking $z=(r, 0, \ldots, 0)^{\prime}(0<r<1)$ in (50) and (51), respectively, we deduce that the equality in (37) holds true. This completes the proof of Theorem 3.

Remark 4. When $n=1$, Theorem 3 reduces to Theorem A.

\section{Conflict of Interests}

The authors declare that there is no conflict of interests regarding the publication of this paper.

\section{Acknowledgments}

This work was supported by NNSF of China (Grant no. 11261022), the Jiangxi Provincial Natural Science Foundation of China (Grant no. 20132BAB201004), the Natural Science Foundation of Department of Education of Jiangxi Province, China (Grant no. GJJ12177), and the Zhejiang Provincial Natural Science Foundation of China (Grant no. Y6110053).

\section{References}

[1] M. Fekete and G. Szegö, "Eine bemerkunguber ungerade schlichte Funktionen," Journal London Mathematical Society, vol. 8, pp. 85-89, 1933.

[2] S. Kanas, "An unified approach to the Fekete-Szegö problem," Applied Mathematics and Computation, vol. 218, no. 17, pp. 8453-8461, 2012.

[3] A. Pfluger, "The Fekete-Szegö inequality for complex parameters," Complex Variables: Theory and Application, vol. 7, no. 1-3, pp. 149-160, 1986.

[4] R. R. London, "Fekete-Szegö inequalities for close-to-convex functions," Proceedings of the American Mathematical Society, vol. 117, no. 4, pp. 947-950, 1993.
[5] B. Bhowmik, S. Ponnusamy, and K. Wirths, "On the FeketeSzegö problem for concave univalent functions," Journal of Mathematical Analysis and Applications, vol. 373, no. 2, pp. 432438, 2011.

[6] L. Bieberbach, "Über die Koeffizienten der einigen Potenzreihen welche eine schlichte Abbildung des Einheitskreises vermitten," S. B. Preuss. Akad. Wiss., vol. 38, pp. 940-955, 1916.

[7] L. de Branges, "A proof of the Bieberbach conjecture," Acta Mathematica, vol. 154, no. 1-2, pp. 137-152, 1985.

[8] H. Cartan, "Sur la possibilité d'étendre aux fonctions de plusieurs variables complexes la théorie des fonctions univalentes," in Lecons sur les Fonctions Univalentes ou Multivalentes, P. Montel, Ed., Gauthier-Villars, Paris, France, 1933.

[9] S. Gong, The Bieberbach Conjecture, American Mathematical Society, International Press, Providence, RI, USA.

[10] I. Graham, H. Hamada, and G. Kohr, "Parametric representation of univalent mappings in several complex variables," Canadian Journal of Mathematics, vol. 54, no. 2, pp. 324-351, 2002.

[11] I. Graham, G. Kohr, and M. Kohr, "Loewner chains and parametric representation in several complex variables," Journal of Mathematical Analysis and Applications, vol. 281, no. 2, pp. 425438, 2003.

[12] H. Hamada, T. Honda, and G. Kohr, "Growth theorems and coefficients bounds for univalent holomorphic mappings which have parametric representation," Journal of Mathematical Analysis and Applications, vol. 317, no. 1, pp. 302-319, 2006.

[13] H. Hamada and T. Honda, "Sharp growth theorems and coefficient bounds for starlike mappings in several complex variables," Chinese Annals of Mathematics B, vol. 29, no. 4, pp. 353-368, 2008.

[14] G. Kohr, "On some best bounds for coefficients of several subclasses of biholomorphic mappings in $C^{n}$," Complex Variables, vol. 36, pp. 261-284, 1998.

[15] X. Liu and T. Liu, "The sharp estimates of all homogeneous expansions for a class of quasi-convex mappings on the unit polydisk in $\mathbb{C}^{n}$," Chinese Annals of Mathematics B, vol. 32, no. 2, pp. 241-252, 2011.

[16] Q. H. Xu and T. S. Liu, "On coefficient estimates for a class of holomorphic mappings," Science in China A: Mathematics, vol. 52, no. 4, pp. 677-686, 2009.

[17] W. Koepf, "On the Fekete-Szegö problem for close-to-convex functions," Proceedings of the American Mathematical Society, vol. 101, no. 1, pp. 89-95, 1987.

[18] T. J. Suffridge, "Starlike and convex maps in Banach spaces," Pacific Journal of Mathematics, vol. 46, pp. 575-589, 1973.

[19] I. Graham and G. Kohr, Geometric Function Theory in One and Higher Dimensions, Monographs and Textbooks in Pure and Applied Mathematics, Marcel Dekker, Inc., New York, 2003. 


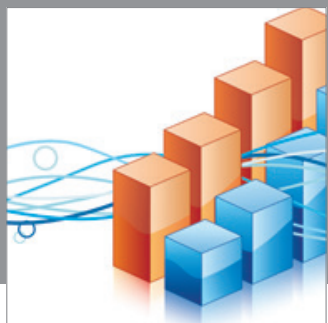

Advances in

Operations Research

mansans

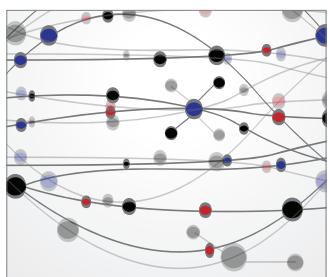

The Scientific World Journal
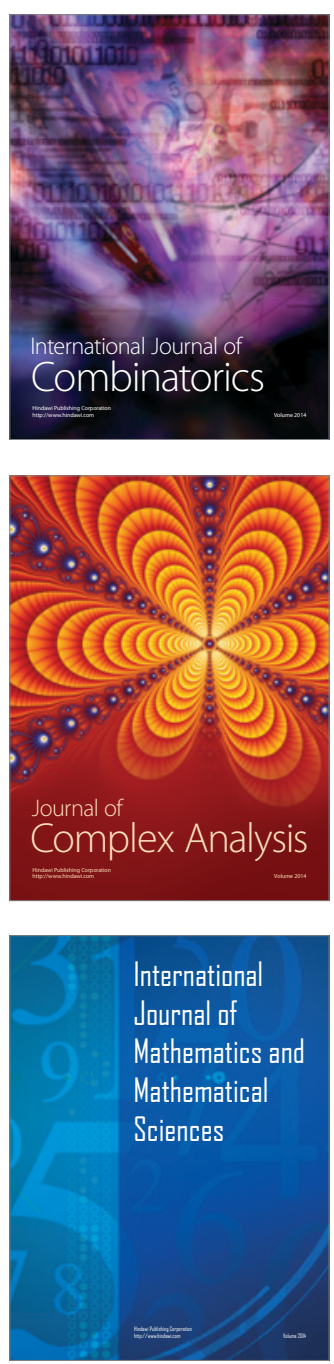
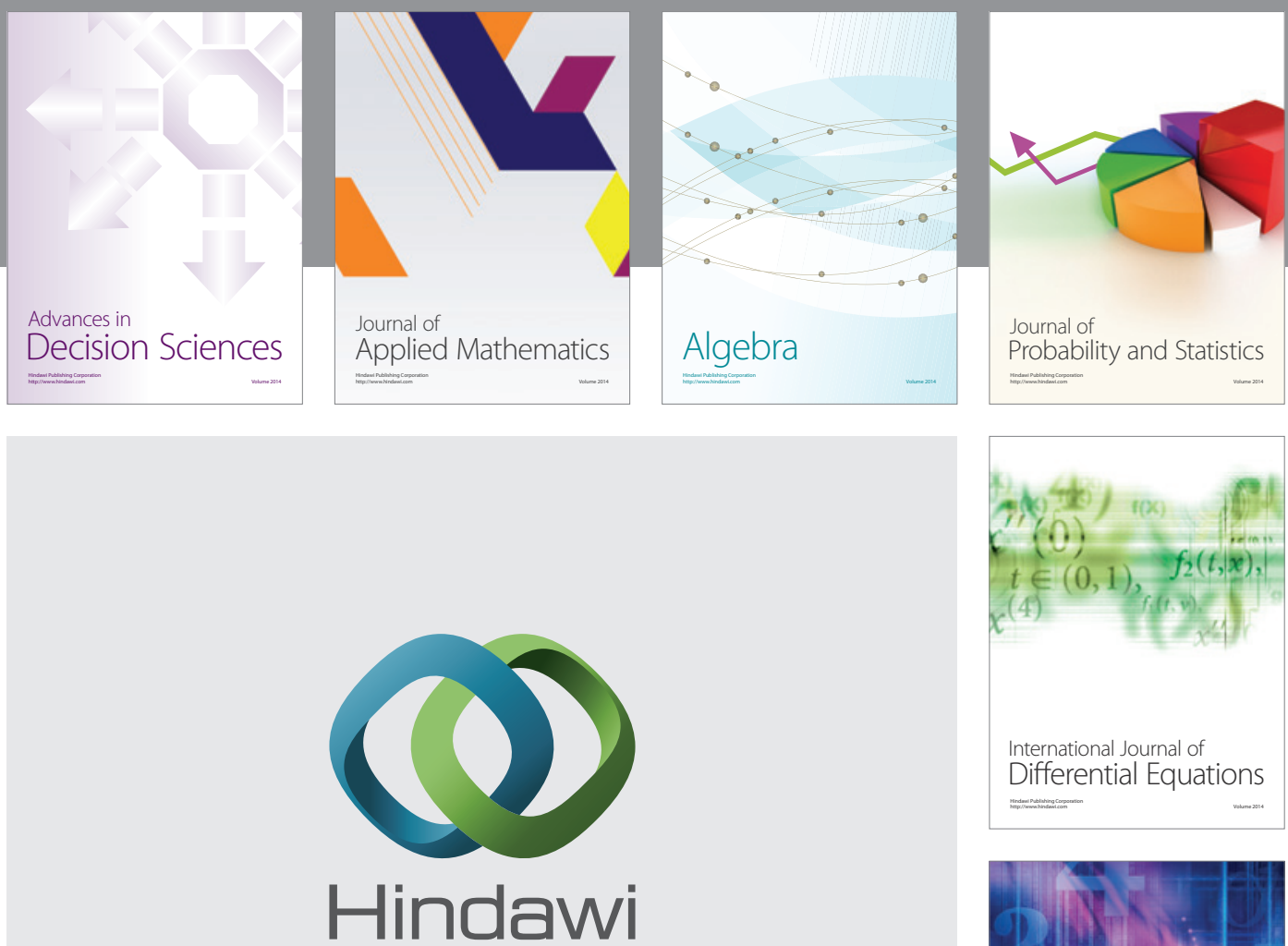

Submit your manuscripts at http://www.hindawi.com
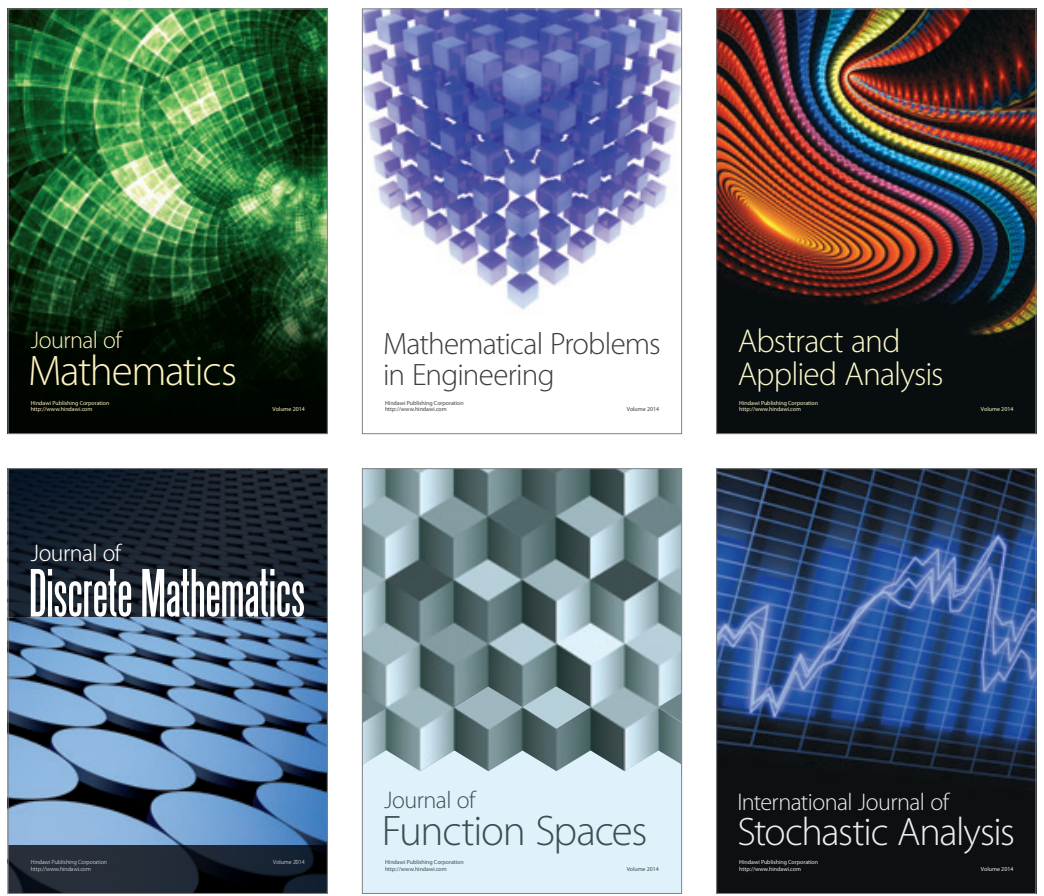

Journal of

Function Spaces

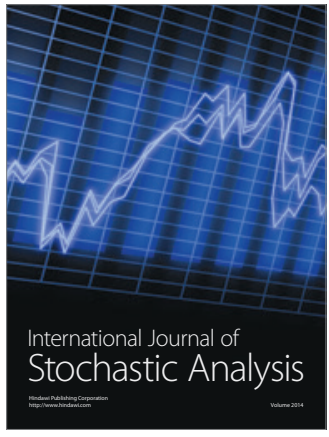

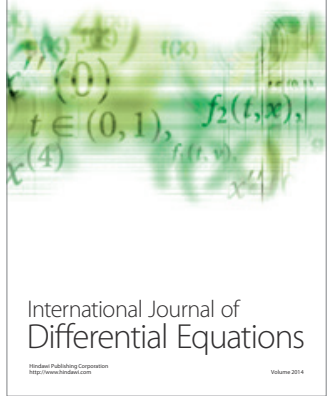
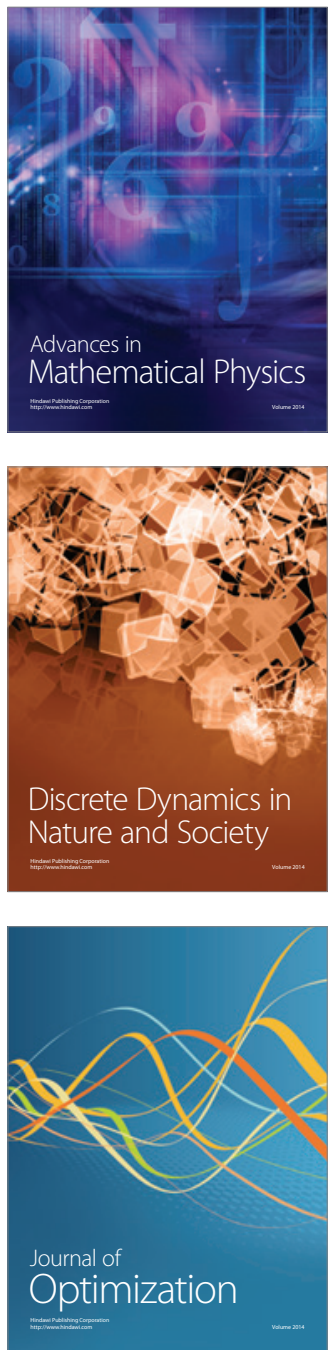